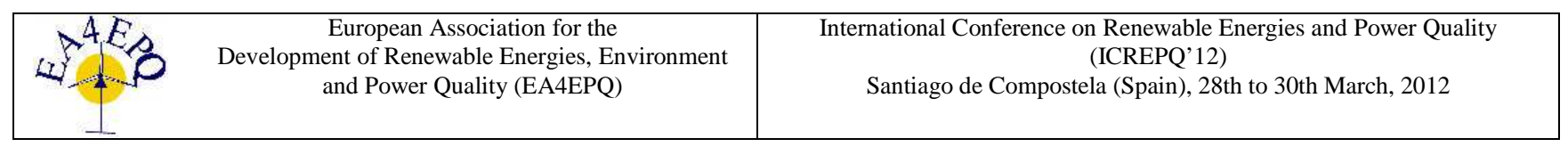

\title{
Impact of Distributed Generation on Fault Locating Methods in Distribution Networks
}

\author{
E. Ebrahimi ${ }^{1}$, A. J. Ghanizadeh ${ }^{1}$, M. Rahmatian ${ }^{1}$ and G. B. Gharehpetian ${ }^{1}$ \\ ${ }^{1}$ Electrical Engineering Department \\ Amirkabir university of Technology \\ Tehran - Iran \\ Phone/Fax number:+982164543504, e-mail: esi_ebrahimi@aut.ac.ir
}

\begin{abstract}
.
In this paper, the effect of inverter based and synchronous DGs on the performance of the impedance based fault locating method is investigated. It is shown that the upstream DGs are more effective in inaccuracy of fault location calculations than downstream DGs.
\end{abstract}

\section{Key words}

Distributed generation (DG), Distribution networks protection, Fault location.

\section{Introduction}

Accurate and precise detection of short circuit fault location is necessary for economical and reliable operation of a power system. Accurate fault location is an important issue for a continuous service in distribution networks, since it reduces the imposed costs of long interruptions for sensitive and industrial loads. Many methods have been presented for fault location determination. They can be divided into two main groups: Methods based on impedance measurement [1-2] and travelling waves generated by short-circuit faults [3-4].

It should be noted that in some papers, expert systems which are mainly based on neural networks have been suggested [5-6]. The algorithms, based on impedance measurement, analyze voltage and current before and during the fault at power frequency. Because of the characteristics of transmission lines length and simplicity of the network, the application of these methods is more usual in transmission systems. These methods act in distribution networks less precisely because of lateral links. Travelling wave methods are based on the measurement of high frequency components of travelling wave and execution of complicated frequency analysis. These high frequency components are generated by shortcircuit fault. Since the generation of these travelling waves is independent from fault type and resistance [7] these methods act precisely, unless the fault occurs at the moment, when the voltage signal is crossing its zero point. In this situation, the generated wave has less amplitude that affects the precision of these methods. Nowadays, the application of these methods in distribution networks has become feasible using Wavelet Transform [3].

The penetration of DGs in distribution networks has changed the radial structure and consequently, the direction and amplitude of the fault current. This phenomenon affects the operation of the impedance method. DG units are connected to the network via inverters or they act through a synchronous generator and are connected to the network directly. In this paper, the effect of both sorts of DG units on the fault locating methods based on the impedance measurement is investigated.

\section{Impedance Measurement Methods}

Impedance Measurement method applies voltage and current signals before and during the fault measured in distribution substation. In this method, the line impedance form the distribution substation up to fault point is calculated and after the comparison between the obtained amount and look-up table, the fault location is determined. The voltage and current signals measured during the fault are passed through a low-pass filter to eliminate their transient terms and just power frequency components are used.

The following stages should be executed to implement the impedance method [8]:

Stage 1) Fault detection: The first step in fault location is the determination of the fault type. At this stage, the rms amount of each phase current is measured at distribution substation and the fault type is determined via comparison of them with a threshold value. Also, the currents of three phases are added and the obtained result is compared with a threshold amount to detect the earth fault. 
Stage 2) Data acquisition: After fault detection, the amount of phase currents and voltages in some cycles before and after fault occurrence are saved. To access power frequency component, the measured signals are passed through a low-pass filter.

Stage 3) Load flow execution: Load flow is executed in distribution network using the data, stored before the fault occurrence, and consequently, the current of each link is obtained. Different methods for the determination of the faulty link in distribution network have been presented. In [9], the following measured difference in distribution substation is presented:

$$
\Delta I=I_{\text {during-fault }}-I_{\text {pre-fault }}
$$

The equality between this difference and the obtained amount for each link's current, expresses the fault on that link. Some other methods apply the comparison of wavelet transform coefficients for the fault location on faulty link [10]. Application of two different frequencies for fault detection has been suggested in [11]. In this paper, the method suggested in [9] will be used.

Stage 4) Extension of the radial network: After detection of the faulty link, all the lateral links will be considered as equivalent impedance at the interconnection point to the main feeder. Using the obtained amounts for voltage and current at stage 2 and considering the fault type, the seen impedance will be calculated.

\section{Basic Calculation}

Suppose, in the network shown in Fig. 1., one phase to ground short-circuit fault has occurred at point F. Zero, positive and negative sequence components of voltage at the fault point are calculated using corresponding values at the beginning of the line and considering voltage drop, as follows:

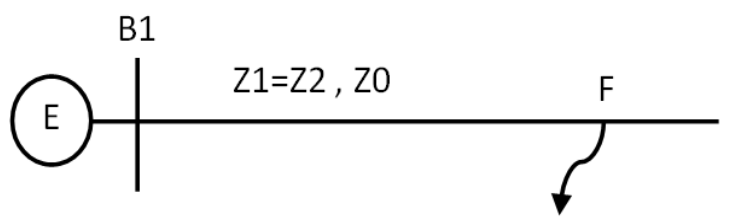

Fig. 1: Single-line diagram of network

$V_{1 F}=V_{1}-I_{1} Z_{1}$

$V_{1 F}=V_{1}-I_{1} Z_{1}$

$V_{0 F}=V_{0}-I_{0} Z_{0}$

where, $\mathrm{Vi}$ is the ith sequence component of voltage at bus $\mathrm{B} 1$, Ii is the ith sequence component of current at bus B1, $\mathrm{ViF}$ is the ith sequence component of voltage at the fault point and $\mathrm{Zi}$ is the impedance of positive, negative and zero sequences for $i=0,1$ and 2 . On the other hand, the sum of the three sequence components of voltage at point $\mathrm{F}$ is proportional to the fault resistance, i. e.:

$V_{1 F}+V_{2 F}+V_{0 F}=3 I_{0} R_{F}$

where, RF is the fault resistance. Sum of the three equations in (1), using (2) and considering $\mathrm{Z} 1=\mathrm{Z} 2$ yields:

$V_{a F}=V_{a}-\left(\left(I_{1}+I_{2}\right) Z_{1}+I_{0} Z_{0}\right)$

After transforming sequential components to fuzzy components, the voltage of phase A (single- phase to ground) can be calculated, as follows:
$V_{a}=\left(I_{a}+k I_{0}\right) Z_{1}+3 I_{0} R_{F}$

$k=\frac{Z_{0}-Z_{1}}{Z_{0}}$

On the other hand, the impedance seen from bus B1 is:

$Z_{\text {app }}=\frac{V_{\text {select }}}{I_{\text {select }}}=R_{\text {app }}+j X_{\text {app }}$

The selected values for single-phase to ground fault is, as follows:

$V_{\text {select }}=V_{a}$

$I_{\text {select }}=\left(I_{a}+k I_{0}\right)=I_{s 1}+j I_{s 2}$

$Z_{\text {app }}=Z_{1}+\frac{3 I_{0} R_{F}}{\left(I_{a}+k I_{0}\right)}$

To compensate the unknown fault resistance, the current injected to the fault should be considered. For this specific case, the compensated current is assumed to be proportional to the zero sequence current:

$Z_{\text {app }}=D z_{1}+\frac{I_{\text {comp }} R_{F}}{\left(I_{a}+k I_{0}\right)}$

where, Icomp is the compensated current, $\mathrm{D}$ is the distance between substation and fault location and $\mathrm{Z} 1$ is the positive sequence impedance of per unit of line length. (9) expresses (8) in matrix form:

$$
\begin{aligned}
& \left(\begin{array}{l}
R_{a p p} \\
X_{a p p}
\end{array}\right)=\left(\begin{array}{c}
R \frac{I_{d} I_{s 1}+I_{q} I_{s 2}}{I_{s}{ }^{2}} \\
X \frac{I_{d} I_{s 2}+I_{q} I_{s 1}}{I_{s}{ }^{2}}
\end{array}\right)\left(\begin{array}{l}
D \\
R_{F}
\end{array}\right) \\
& I_{c o m p}=I_{d}+j I_{q} \\
& I_{s}{ }^{2}=I_{s 1}{ }_{s 1}+I_{s 2}
\end{aligned}
$$

Using (9), the distance between the fault and bus B1 is calculated, as follows:

$$
\begin{aligned}
& D=\frac{\left(R_{a p p} M-X_{a p p} L\right)}{\left(R_{1} M-X_{1} L\right)} \\
& L=\frac{I_{d} I_{s 1}+I_{q} I_{s 2}}{I_{s}{ }^{2}} \\
& M=\frac{I_{d} I_{s 2}+I_{q} I_{s 1}}{I_{s}{ }^{2}}
\end{aligned}
$$

The aforementioned equations are applicable for different fault types. Of course, the selected values for current, voltage and compensated current will be different for various fault types [12].

\section{Effect of DG on Impedance Measurement Based Method}




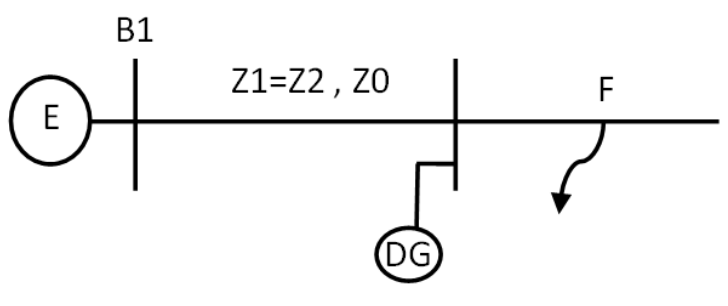

Fig. 2: DG unit placed at upstream

B1

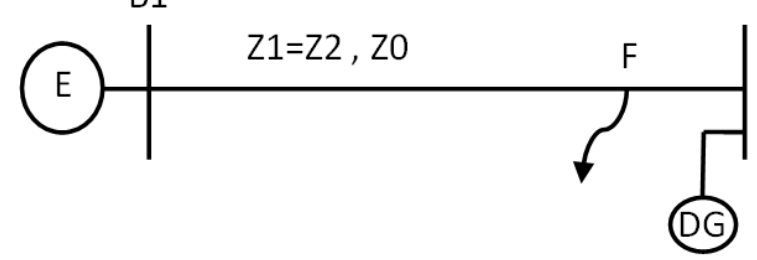

Fig. 3: DG unit placed at downstream

To investigate DG effect, first assume the fault has occurred at the downstream of the DG as depicted in Fig. 2. In this situation, the fault current at point $F$ is supplied by the network and DG unit. Therefore, the effect of DG in the mentioned equations will appear, as below:

$$
\begin{aligned}
V_{\text {select }} & =V_{a} \\
I_{\text {select }} & =\left(\left(I_{a}+I_{a-D G}\right)+k\left(I_{0}+I_{0-D G}\right)\right) \\
& =I_{s 1-\text { new } 1}+j I_{s 2-\text { new } 1} \\
I_{\text {comp }} & =3\left(I_{0}+I_{0-D G}\right)=I_{d-\text { new } 1}+j I_{q-\text { new } 1}
\end{aligned}
$$

By substituting (14) and (15) in (9) to (13), the obtained result for the fault location distance $\mathrm{D}$, will be different from the situation, in which DG unit is not present. In other words, the amount of the fault current injected by DG is unknown and the fault location is just possible using the measured parameters at the distribution substation. Hence, the fault locating method precision with the presence of DG will reduce.

Now, assume that the DG unit is located at downstream of the fault point according to Fig. 3 .

In this case, just the amount of zero sequence component of fault current of DG appears in the equations, therefore, we have;

$$
\begin{aligned}
& V_{\text {select }}=V_{a} \\
& I_{\text {select }}=I_{a}+k\left(I_{0}+I_{0-D G}\right)=I_{s 1-\text { new } 1}+j I_{\text {s2-new } 2} \\
& I_{\text {comp }}=3\left(I_{0}+I_{0-D G}\right)=I_{d-\text { new } 1}+j I_{q-\text { new } 1}
\end{aligned}
$$

In this situation, the amount of zero sequence component of the DG fault current in distribution substation is unknown.

Consequently, the calculations for fault location are not precise. Because of nominal values of semi-conductor switches, the inverter based DG units are not able to tolerate huge amounts of short circuit current. Therefore, the effect of them on fault location inaccuracy is less than synchronous DGs.

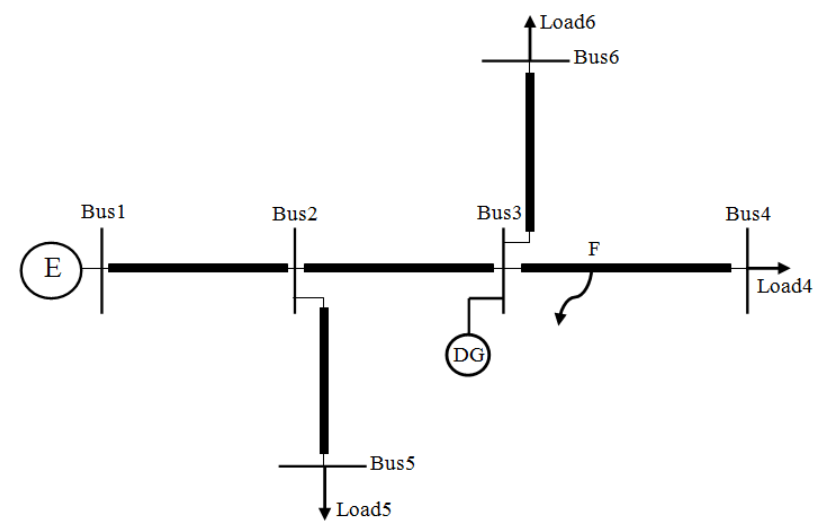

Fig. 4: Distribution network with DG at upstream

\section{Simulation Results}

To investigate the effect of DGs on distribution networks, the aforementioned algorithms are tested on the network shown in Fig. 4. The impedance of per unit length of lines is $0.3 \Omega / \mathrm{km}$ and their length is listed in Table I.

Table I: Length of distribution network lines

\begin{tabular}{|c|c|c|c|c|c|}
\hline Bus & $1-2$ & $2-3$ & $3-4$ & $2-5$ & $3-6$ \\
\hline Length $(\mathrm{km})$ & 2 & 3 & 5 & 1 & 2 \\
\hline
\end{tabular}

An inverter based and a synchronous DG are selected with the capacity of $5 \mathrm{MW}$ and $30 \mathrm{MW}$, respectively. The amount of loads connected to bus bars is listed in Table II. As the first case, assume that the DG unit is connected to bus 3 and single-phase to ground fault is occurred at point $\mathrm{F}$ between buses 3 and 4 .

Table II: Loads connected to distribution network

\begin{tabular}{|c|c|c|}
\hline $\begin{array}{c}\text { Consumption } \\
\text { bus }\end{array}$ & $\mathrm{P}(\mathrm{MW})$ & $\mathrm{Q}(\mathrm{kVAR})$ \\
\hline Bus 4 & 3.6 & 12.5 \\
\hline Bus 5 & 7.5 & 42.5 \\
\hline Bus 6 & 7.5 & 42.5 \\
\hline
\end{tabular}

Table III gives the calculation error for fault location with the presence of DG unit considering different locations for fault. It is obvious that the calculations precision has reduced with the presence of DG unit. It should be noted that the calculation error is obtained according to (18) [13]:

$\operatorname{Error}(\%)=\frac{\operatorname{Re} a l D-C a l D}{\text { TotalD }} \times 100$

where, RealD is the real distance of the short-circuit fault, CalD is the calculated distance and TotalD is the total line length.

To investigate the effect of downstream DG of the fault, assume that the DG unit is connected to bus 4 according to Fig. 5. As mentioned before, in this case, just the zero sequence component of DG fault current is important for calculations and the amount of the calculation error is less than the previous case. These results are presented in Table IV. 
Table III: Calculation error with DG at upstream of fault

\begin{tabular}{|c|c|c|c|c|}
\hline & \multicolumn{3}{|c|}{ Fault location from bus $1(\mathrm{~km})$} \\
\hline & & Real & Calculated & $\begin{array}{c}\text { Calculation } \\
\text { error }(\%)\end{array}$ \\
\hline \multirow{9}{*}{$\begin{array}{c}\text { DG at } \\
\text { downstream } \\
\text { of fault }\end{array}$} & \multirow{3}{*}{$\begin{array}{c}\text { Without } \\
\text { presence of } \\
\text { DG } \\
\end{array}$} & 7 & 7.020 & 0.2 \\
\hline & & 8 & 8.260 & 2.6 \\
\hline & & 9 & 9.497 & 4.97 \\
\hline & \multirow{3}{*}{$\begin{array}{c}\text { Synchronous } \\
\text { DG }\end{array}$} & 7 & 7.293 & 2.93 \\
\hline & & 8 & 8.677 & 6.77 \\
\hline & & 9 & 10.050 & 10.5 \\
\hline & \multirow{3}{*}{$\begin{array}{c}\text { Inventor } \\
\text { Based DG }\end{array}$} & 7 & 7.167 & 1.67 \\
\hline & & 8 & 8.483 & 4.83 \\
\hline & & 9 & 9.780 & 7.8 \\
\hline
\end{tabular}

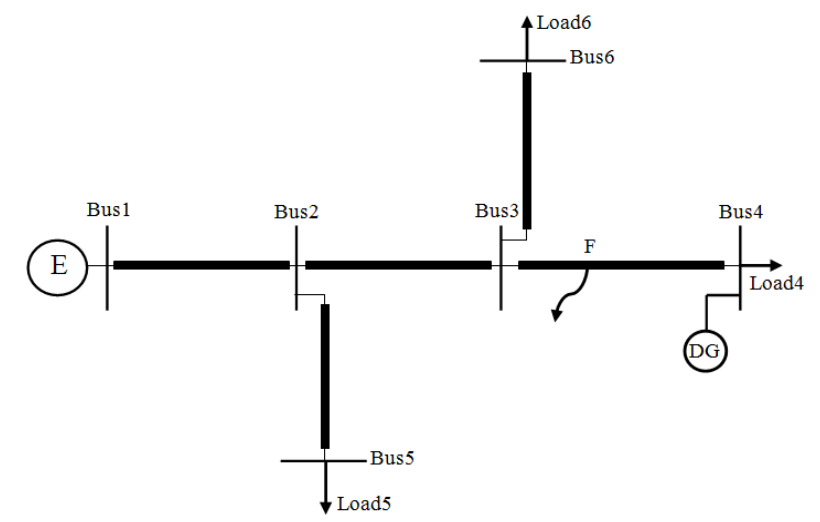

Fig. 5: Distribution network with DG unit at downstream of fault

For an accurate perception of DG unit effect, assume that it has been installed at bus 3 and single-phase to ground fault has occurred at different points between buses 1 and 4 . In this case, DG unit is placed at downstream for faults between buses 1 and 3 and in contrast, it is at upstream for faults between buses 3 and 4 .

Table IV: Calculation error with presence of DG at downstream of fault

\begin{tabular}{|c|c|c|c|c|}
\hline & \multicolumn{3}{|c|}{ Fault location from bus $1(\mathrm{~km})$} \\
\hline & & Real & Calculated & $\begin{array}{c}\text { Calculation } \\
\text { error }(\%)\end{array}$ \\
\hline \multirow{9}{*}{$\begin{array}{c}\text { DG at } \\
\text { downstream } \\
\text { of fault }\end{array}$} & \multirow{3}{*}{$\begin{array}{c}\text { Without } \\
\text { presence of } \\
\text { DG } \\
\end{array}$} & 7 & 7.020 & 0.2 \\
\hline & & 8 & 8.260 & 2.6 \\
\hline & & 9 & 9.497 & 4.97 \\
\hline & \multirow{3}{*}{$\begin{array}{c}\text { Synchronous } \\
\text { DG }\end{array}$} & 7 & 7.080 & 0.8 \\
\hline & & 8 & 8.347 & 3.47 \\
\hline & & 9 & 9.607 & 6.07 \\
\hline & \multirow{3}{*}{$\begin{array}{l}\text { Inventor } \\
\text { Based DG }\end{array}$} & 7 & 7.050 & 0.5 \\
\hline & & 8 & 8.310 & 3.1 \\
\hline & & 9 & 9.560 & 5.6 \\
\hline
\end{tabular}

Figure 6 expresses the calculated errors originated from the presence of the DG unit. Considering the figure, it is clear that at the points near the distribution bus, calculation error originated from the presence of DG unit is almost equal to the case of its absence. This phenomenon occurs since; in this case, most of the short circuit current is injected by the distribution network. In contrast, for the faults between buses 3 and 4, the calculation error is considerable, since DG unit is at

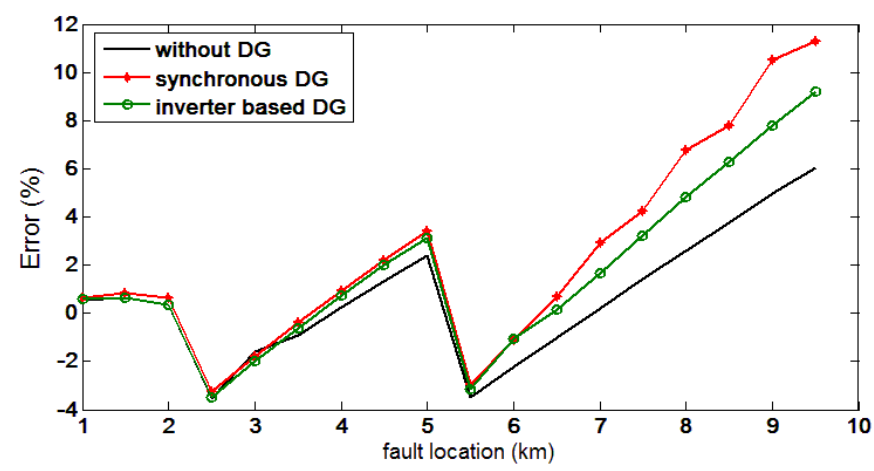

Fig. 6: Calculation error originated from inventor-based and synchronous DGs for upstream and downstream faults versus fault distance form bus1

upstream of the fault. When the fault approaches bus 4, the impedance in front of the DG unit grows. Hence, calculation error increases. On the other hand, since the DG unit is located at downstream for the faults between buses 1 and 3 , calculation error originated from the presence of DG is less than the cases, in which the DG unit has been placed at upstream. Also, it is clear that calculation error for the inverter-based DG is less than the synchronous DG.

\section{Conclusions}

Considering the participation of DG units in fault currents, the operation of fault locating methods based on impedance measurement can be affected. In this paper, the effect of two types of DGs on the fault location has been investigated. Inventer-based DGs inject less short-circuit current to the fault. Therefore, they are less effective in malfunction of impedance based methods in comparison to synchronous DGs. Also, it has been shown that upstream DGs have more negative effects on fault locating methods in comparison to downstream DGs.

\section{References}

[1] Myeon-Song Choi, Seung-Jae Lee, Seong-Il Lim, Duck-Su Lee, and Xia Yang "A Direct Three-Phase Circuit AnalysisBased Fault Location for Line-to-Line Fault" IEEE Trans. Power Del., Vol. 22, No. 4, October 2007.

[2] Karl Zimmerman and David Costello, Schweitzer “ Impedance-Based Fault Location Experience" 58th Annual Conference for Protective Relay Engineers, pp. 211-226, 2005.

[3] Alberto Borghetti, Mauro Bosetti, Mauro Di Silvestro, Carlo Alberto Nucci, and Mario Paolone " Continuous Wavelet Transform for Fault Location in Distribution Power Networks: Definition of Mother Wavelets Inferred From Fault Originated Transients" IEEE Trans. Power Syst., Vol. 23, No. 2, May 2008.

[4] M.da Silva D.V. Coury M. Oleskovicz E.C. Segatto “ Combined solution for fault location in three terminal lines based on wavelet transforms" IET ,Generation, Transmission \& Distribution, pp. 94-103, 2010.

[5] S. Ebron, D. L. Lubkeman, and M. White, "A neural network approach to the detection of incipient faults on power distribution feeders" IEEE Trans. Power Del., vol. 5, no. 2, pp. 905-914, Apr. 1990. 
[6] Z. Chen and J. C. Maun, "Artificial neural network approach to single ended fault locator for transmission lines" IEEE Trans. Power Syst., vol. 15, No. 1, pp. 370-375, Feb. 2000.

[7] F. H. Magnago and A. Abur, "Fault location using wavelets," IEEE Trans. Power Del., Vol. 13, No. 4, pp. 1475-1480, Oct. 1998.

[8] Sachdev, M.S.; Das, R.; Sidhu, T.S "Determining locations of faults in distribution systems" International Conference on Developments in Power System Protection, pp. 188-191, 1997.

[9] Mohammad A. Mirzai and Ali A. Afzalian "A Novel FaultLocator System; Algorithm, Principle and Practical Implementation" IEEE Trans. Power Del., Vol. 25, No. 1, January 2010.

[10] Fernando E-I. Magnago and Ali Abur "A New Fault Location Technique for Radial Distribution Systems Based on High Frequency Signals" Power Engineering Society Summer Meeting, IEEE, Vol. 1, pp. 426 - 431, 1999.

[11] Fengling Han, Xinghuo Yu, Majid Al-Dabbagh and Yi Wang "Locating Phase to Ground Short Circuit Faults on Radial Distribution Lines" IEEE Trans industrial electronics, Vol. 54, No. 3, June 2007.

[12] Adly A. Girgis, Christopher M. Fallon, and David L. Lubkeman "A Fault Location Technique for Rural Distribution Feeders" IEEE Trans industrial applications, Vol. 29, No. 6, November 1993.

[13] Seung-Jae Lee, Myeon-Song Choi,Sang-Hee Kang,Bo-Gun Jin, Duck-Su Lee, Bok-Shin Ahn,Nam-Seon Yoon, Ho-Yong Kim, and Sang-Bong Wee "An Intelligent and Efficient Fault Location and Diagnosis Scheme for Radial Distribution Systems" IEEE Trans. Power Del., Vol. 19, No. 2, April 2004. 